\title{
Long- and short-term outcomes of early gastric cancer after endoscopic resection: a retrospective study from China
}

\section{다)(1) $\odot$}

\author{
Authors \\ Institutions \\ 1 Division of Gastroenterology and Hepatology, Key \\ Laboratory of Gastroenterology and Hepatology, \\ Ministry of Health, Renji Hospital, School of Medicine, \\ Shanghai Jiao Tong University, Shanghai Institute of \\ Digestive Disease, Shanghai, China \\ 2 Department of Gastroenterology and Hepatology, \\ Chongqing Hospital of Traditional Chinese Medicine, \\ Chongqing, China
}

Qing-Wei Zhang*,, Jin-Nan Chen ${ }^{*, 1}$, Zhao-Rong Tang*,2, Yun-Jie Gao', Zhi-Zheng Ge**, ${ }^{*}$, Xiao-Bo Li ${ }^{* *, 1}$

submitted 17.2.2020

accepted after revision 18.12 .2020

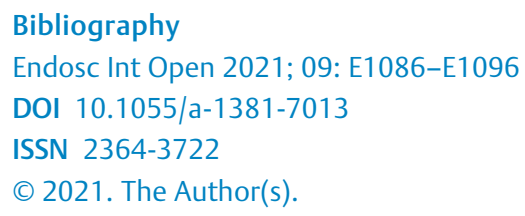
Commons Attribution-NonDerivative-NonCommercial License, permitting copying and reproduction so long as the original work is given appropriate credit. Contents may not be used for commercial purposes, or adapted, remixed, transformed or built upon. (https://creativecommons.org/licenses/by-nc-nd/4.0/)

Georg Thieme Verlag KG, Rüdigerstraße 14,

70469 Stuttgart, Germany

Corresponding author

Xiao-Bo Li, Shanghai Jiao Tong University School of Medicine Affiliated Renji Hospital - Department of Gastroenterology, Pujian Road 160, Shanghai 200127, China

Fax: +021-68383015

Ixb_1969@163.com

\author{
丹upplementary material is available under \\ https://doi.org/10.1055/a-1381-7013
}

\section{ABSTRACT}

Background and study aims The aim of the study was to evaluate short- and long-term outcomes of endoscopic submucosal dissection (ESD) for early gastric cancer (EGC) in China because no study has yet been conducted to confirm its effectiveness in EGC in China.

Patients and methods A total of 570 EGC samples from 537 patients were collected for evaluation of en bloc, complete, and curative resection using ESD. Data from 302 patients with at least 3 years of active follow-up were collected for analysis of recurrence of EGC and occurrence of metachronous GC (MGC). Short- and long-outcomes of mixedtype and pure differentiated EGC were also compared.

Results En bloc resection rates of $96.0 \%, 98.7 \%$, and $95.2 \%$, complete resection rates of $91.2 \%, 96.6 \%$ and $90.8 \%$, and curative resection rates of $83.0 \%, 96.2 \%$ and $88.2 \%$ were achieved in all EGCs included in the study, those with absolute indication, and those with expanded indication, respectively. As a long-term outcome, recurrence was observed in $1.3 \%$ of patients, 3 -year and 5 -year recurrence rates being $0.7 \%$ and $1.2 \%$, respectively. Thirteen patients (4.3\%) exhibited MGCs during follow-up, all of which were resected in a second ESD.

Conclusions The effectiveness of ESD for EGC in China was confirmed, with satisfactory short- and long-term outcomes. With scheduled follow-up, the outcomes for mixed-type EGC can be similar to those for pure differentiated EGC after complete resection without development of lymphovascular invasion.

\section{Introduction}

With the introduction of a national screening policy in Asian countries and advancements in endoscopic technology, a greater number of early gastric cancers (EGCS) have been de-

\footnotetext{
* These authors contributed equally.

** These authors contributed equally.
}

tected, with 5-year survival reaching more than $90 \%[1,2]$. Endoscopic submucosal dissection (ESD), an advanced endoscopic resection technique, has been widely accepted as the primary treatment of choice for EGC [3-6] because of the low prevalence of lymph node metastasis (LNM) in EGC $[7,8]$, thus avoiding unnecessary gastrectomy following what is considered curative resection based on histological examination of ESD specimens [9]. 
Since the development of ESD more than 10 years ago, multiple studies have investigated short- and long-term outcomes of ESD of EGC [1-6] with favorable outcomes compared with surgery or endoscopic mucosal resection (EMR). However, there remains limited evidence about short-term outcomes of ESD of EGC [10-13], with only small sample sizes in China. Although it has been reported that a 3-year survival rate of 94.6\% was achieved in ESD groups involving only 74 lesions, there still remains a lack of data reporting the recurrence of EGC or occurrence of metachronous GC in China that allows evaluation of long-term outcomes of ESD and whether the effectiveness of endoscopic surveillance using Japanese guidelines is appropriate for local conditions [9].

In addition, recent studies have shown that EGCs with mixed differentiated and undifferentiated components (mixed-type) exhibit a higher risk of LNM compared with pure differentiated EGC [14-16] and even higher than pure undifferentiated EGC $[15,16]$. Although a number of studies have been conducted that have investigated effectiveness and long-term outcomes of ESD for therapy of mixed-type EGCs [17-19], contradictory results have been observed. Therefore, additional studies are urgently required to investigate whether ESD is suitable for mixed-type EGCs and to establish appropriate indications for it.

Therefore, this first study to assess short- and long-term outcomes of ESD for EGC was conducted based on data from a large consecutive patient series involving 570 EGCs in China. Furthermore, we attempted to compare short- and long-term outcomes of ESD among patients with pure differentiated or pure undifferentiated EGC and patients with mixed-type EGC.

\section{Patients and methods}

\section{Patients}

In the present study, patients with histologically confirmed EGCs undergoing ESD in Shanghai Renji Hospital from 2008 to 2018 were included to assess the short-term outcomes of ESD. Meanwhile, patients diagnosed between 2008 and 2015 were also analyzed to assess long-term outcomes in patients with EGC following ESD.

Patients were excluded if they fulfilled the following criteria: 1) Final pathology of the lesion was not classified as epithelial malignancy; 2) they had other cancers; or 3) they had received chemotherapy or radiotherapy prior to ESD.

All patients included in the study had provided written informed consent prior to ESD of their EGC. Approval for the study was granted by the institutional review board at Renji Hospital.

\section{ESD procedure}

All ESD procedures were performed by skilled and trained endoscopists. First, after careful visualization of the lesion margins, the clinician used argon plasma coagulation (APC) to create a circumferential mark $5 \mathrm{~mm}$ from the outer edge of the lesion. Second, a mixture of hyaluronic acid, $10 \%$ glycerol plus $5 \%$ fructose in $0.9 \%$ saline or $20 \%$ glucose (containing $0.005 \%$ indigo carmine and $0.0005 \%$ epinephrine) was injected into the submucosal layer of the lesion to form a submucosal fluid cush- ion. Third, an initial incision was created using an electrosurgical dual knife (KD-650Q; Olympus) outside the mark to separate the lesion from the surrounding healthy mucosa. Fourth, either a dual knife (KD-650Q; Olympus) or IT Knife2 (Olympus Medical Systems) was used to dissect the submucosal layer and finally remove the lesion. During the ESD procedure, hemostatic forceps (HDB2422W; Pentax, Tokyo, Japan) were used to control bleeding during the procedure or for ablation of visible vessels on the mucosal defect after resection.

\section{Histopathological evaluation}

After resection of the EGC, histopathological assessment was performed in accordance with the recommendations of the Japanese Gastric Cancer Association [20]. All ESD specimens was sectioned serially at $2 \mathrm{~mm}$ intervals parallel to a line closest to the resection margin so as to effectively assess horizontal and vertical margins after fixation of the specimens. Tumor size, histopathology, ulceration (UL), horizontal margin (HM), vertical margin (VM), invasion depth and lymphovascular invasion (LVI) were assessed for each ESD specimen. Tumors that had invaded the submucosa was subclassified as pSM1 $(<500 \mu \mathrm{m}$ from the muscularis mucosae) or pSM2 (>500 $\mu \mathrm{m}$ from the muscularis mucosae) $[9,20]$. Positive LVI was finally determined using immunohistochemistry of suspected lesions with positive LVI in hematoxylin and eosin-stained sections using the monoclonal antibody D2-40 and/or Victoria blue or Elastica van Gieson staining. Ulcer was defined histologically as a disruption of the muscularis mucosae with or without granulation tissue formation or submucosal fibrosis. Histology of the lesions was classified as differentiated adenocarcinoma, undifferentiated adenocarcinoma or mixed-type adenocarcinoma [20,21]. Differentiated adenocarcinomas consist of papillary adenocarcinoma, or well or moderately differentiated adenocarcinoma. Undifferentiated adenocarcinoma includes poorly differentiated adenocarcinoma, signet-ring cell adenocarcinoma and mucinous adenocarcinoma. Mixed-type adenocarcinoma is defined as adenocarcinoma with components of differentiated and undifferentiated adenocarcinoma according to the Korean and Japanese guidelines [19-21]. In the present study, mixedtype EGC was defined as either mixed predominantly differentiated (MD) type or mixed predominantly undifferentiated (MU) type [16]. All histological examinations of EGC were determined by expert pathologists.

\section{Pathological curability}

According to the 2010 Japanese treatment guidelines for gastric cancer [9], complete ESD is defined as en bloc resection with negative horizonal and vertical margins. Curative ESD is defined, in terms of absolute indication, as en bloc resection for mucosal $(\mathrm{M})$ differentiated-type tumors $\leq 2 \mathrm{~cm}$ with negative HM, negative VM, and no LVI. The expanded indication is defined as a tumor fulfilling one of the following criteria: (1) Differentiated type, pM, UL (-), size $>2 \mathrm{~cm}$; (2) Differentiated, pM, UL $(+)$, size $\leq 3 \mathrm{~cm}$; (3) Differentiated, pSM1, UL $(-), \leq$ $3 \mathrm{~cm}$; (4) Undifferentiated, pM, UL (-), $\leq 2 \mathrm{~cm}$. Therefore, curative ESD is defined, using the expanded indications, as en bloc 
resection for EGCs fulfilling one of the expanded indications without positive HM, VM or LVI. ESD with histological assessment not fulfilling the above-mentioned curative ESD criteria was considered noncurative ESD. For EGC with mixed adenocarcinoma, pathological curability was assessed where MD-EGC was considered to be differentiated EGC and MU-EGC was considered to be undifferentiated EGC.

\section{Complications}

Bleeding was confirmed by endoscopy when symptoms of melena, hematochezia, or hematemesis were recorded following ESD. Perforation was diagnosed by observation of mesenteric fat on gastroscopy or free air on radiography examination.

\section{Follow-up after ESD}

All patients were systematically followed-up in accordance with a defined follow-up procedure, by which patients were asked to attend hospital after contact using phone or Wechat. Patients treated with curative ESD were followed-up using endoscopy examination and abdominal computed tomography (CT) every 6 to 12 months. For patients treated with noncurative ESD, patients at possible risk of LNM were recommended to have additional surgery with lymph node dissection [9]. In addition, abdominal CT or abdominal ultrasonography and endoscopy was performed every 12 months in noncurative ESD patients undergoing additional gastrectomy. Patients with noncurative ESD who did not undergo surgical resection, were followed-up endoscopically with additional abdominal CT, abdominal ultrasonography, or endoscopic ultrasonography every 6 to 12 months for at least 3 years, with an annual follow-up thereafter. Long-term outcome data describing recurrence or development of metachronous adenocarcinoma were collected for each patient. Recurrence was defined as recurrent adenocarcinoma observed at the site of the prior ESD. Metachronous gastric cancer (MCC) was defined as adenocarcinoma detected in the stomach at a site different from that of the prior ESD after 12 months.

\section{Statistical analysis}

Absolute numbers were used to present descriptive statistics, proportions were used for categorical variables, means and standard deviation for continuous variable having a normal distribution, and median and interquartile range (IQR) for continuous variables having a non-normal distribution, respectively. A chi-square test was conducted for categorical variables, student's $t$-test for continuous variables with a normal distribution and a nonparametric Kruskal-Wallis rank sum test for continuous variables with a non-normal distribution, to compare different patient groups, respectively. A multivariate logistic regression model was performed to identify independent risk factors for outcomes of interest.

Time of recurrence or development of MGC was calculated as the interval between date of first ESD and date of outcomes of interest or the last date when patients underwent endoscopy or CT scan. Survival curves were plotted using a Kaplan-Meier method and analyzed using a log-rank test. A multivariate Cox regression model was used to explore risk factors for recurrence.

Statistical analyses and plotting graphics were conducted using the R software package (version R-3.4.3, the R Foundation for statistical computing). All statistical comparisons were considered significant where $P<0.05$.

\section{Results}

\section{Clinical Characteristics of patients and lesions}

As shown in - Fig. 1, a total of 570 EGC samples from 537 patients were included in the present study for analysis of shortterm outcomes after ESD. In addition, 302 patients with at least 3 years of active follow-up from 2008 to 2015 were included for analysis of long-term outcomes after ESD.

Median patient age was 64 years (IQR: 58,70$)$. A total of 520 EGCs (91.2\%) were diagnosed as differentiated, $13(2.3 \%)$ as undifferentiated and $37(6.5 \%)$ as mixed-type, consisting of 11 MU-EGCs (1.9\%) and 26 MD-EGCs (4.6\%). Furthermore, 32 le-

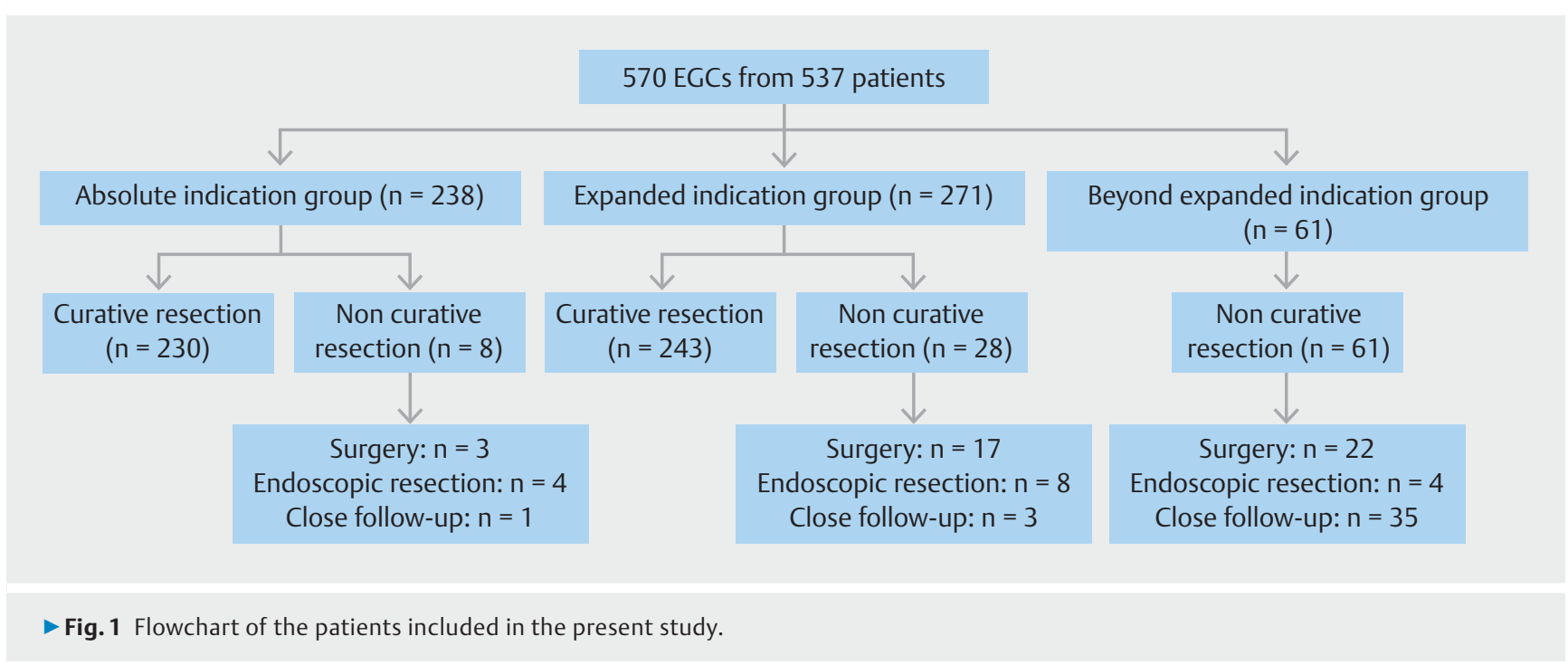


- Table 1 Patient and lesion characteristics.

\begin{tabular}{|c|c|}
\hline \multicolumn{2}{|l|}{ Characteristics } \\
\hline Age (year) & $64(58,70)$ \\
\hline \multicolumn{2}{|l|}{ Sex } \\
\hline - Male & $367(68.3 \%)$ \\
\hline - Female & $170(31.7 \%)$ \\
\hline Size $(\mathrm{mm})$ & $20.0(11.3,28.0)$ \\
\hline \multicolumn{2}{|l|}{ Location } \\
\hline - Antrum & $273(47.9 \%)$ \\
\hline - Angle & $100(17.5 \%)$ \\
\hline - Body & $100(17.5 \%)$ \\
\hline - Fundus or cardia & $97(17.0 \%)$ \\
\hline \multicolumn{2}{|l|}{ Macroscopic type } \\
\hline - Depressed & $143(25.1 \%)$ \\
\hline - Elevated & $164(28.8 \%)$ \\
\hline - Flat & $38(6.7 \%)$ \\
\hline - Mixed & $219(38.4 \%)$ \\
\hline - Undetermined & $6(1.0 \%)$ \\
\hline \multicolumn{2}{|l|}{ History of surgery } \\
\hline - No surgery & $562(98.6 \%)$ \\
\hline - Gastrectomy & $7(1.2 \%)$ \\
\hline - Esophagectomy & $1(0.2 \%)$ \\
\hline \multicolumn{2}{|l|}{ Histology } \\
\hline \multicolumn{2}{|l|}{ Differentiated } \\
\hline - Pap & $3(0.5 \%)$ \\
\hline " Tub1 & $499(87.5 \%)$ \\
\hline " Tub2 & $18(3.2 \%)$ \\
\hline \multicolumn{2}{|l|}{ Undifferentiated } \\
\hline " Por & $3(0.5 \%)$ \\
\hline . Sig & $8(1.4 \%)$ \\
\hline " Muc & $2(0.4 \%)$ \\
\hline \multicolumn{2}{|l|}{ Mixed } \\
\hline . MD & $11(1.9 \%)$ \\
\hline . MU & $26(4.6 \%)$ \\
\hline \multicolumn{2}{|l|}{ Depth } \\
\hline " $\mathrm{pM}$ & $494(86.7 \%)$ \\
\hline - pSM1 & $43(7.5 \%)$ \\
\hline - pSM2 & $32(5.6 \%)$ \\
\hline Undetermined & $1(0.2 \%)$ \\
\hline \multicolumn{2}{|l|}{ LVI status } \\
\hline . No & $553(97.0 \%)$ \\
\hline - Yes & $16(2.8 \%)$ \\
\hline
\end{tabular}

- Table 1 (Continuation)

\section{Characteristics}

- Undetermined

$1(0.2 \%)$

UL

- No

$556(97.5 \%)$

- Yes

$14(2.5 \%)$

Pap, papillary adenocarcinoma; Tub1, well-differentiated tubular adenocarcinoma; Tub2, moderately differentiated tubular adenocarcinoma; Por, poorly differentiated adenocarcinoma; Sig, signet ring cell carcinoma; Muc, mucinous adenocarcinoma; pM, intramucosal; PSM1, depth of invasion < 500 um from the muscularis mucosae; pSM2, depth of invasion $\geq 500$ um from the muscularis mucosae; LVI, lymphatic vessel infiltration; UL, ulcer.

sions (5.6\%) were diagnosed as PSM2 EGCs and 16 EGCs (2.8\%) had positive LVI. Detailed information about the included EGCS is described in $>$ Table 1 .

\section{Pathological curability}

As shown in > Table 2, in patients undergoing ESD, 238 (41.8\%) EGCs fulfilled the absolute indication and 271 (47.5\%) an expanded indication, including 236 (87.1\%) differentiated mucosal EGCs without ulceration, three (1.1\%) differentiated mucosal EGCs $<30 \mathrm{~mm}$ with ulceration, 25 (9.2\%) differentiated pSM1 EGCs < $30 \mathrm{~mm}$ without ulceration and seven (3.0\%) undifferentiated mucosal EGCs $<20 \mathrm{~mm}$ without ulceration.

En bloc resection was performed in 547 EGCs (96.0\%), including 235 (98.7\%) in 238 EGCs with absolute indication and 258 (95.2\%) in 271 EGCs with expanded indications. Based on histopathology and assessment of margins, complete ESD was achieved in 520 EGCs (91.2\%), including 230 EGCs (96.6\%) with absolute indication and 246 EGCs (90.8\%) with an expanded indication. Curative ESD was achieved in 473 EGCs (83.0\%), including 229 EGCs (96.2\%) with absolute indication and 239 (88.2\%) with an expanded indication. Noncurative ESD was observed in 96 EGCs (16.8\%) with 47 lesions (49.0\%) having positive resection margins, 16 lesions (17.7\%) with positive LVI and 61 (63.5\%) beyond expanded indications. In addition, nine (1.5\%) tried ESD but not completed. For patients with noncurative resection, 39 patients (40.2\%) were closely followed-up without any subsequent treatment following ESD, 42 patients (43.3\%) underwent surgery after ESD and 16 (16.5\%) underwent repeated endoscopic resection.

The histological characteristics and short-term outcomes of ESD were next explored according to different histological types. As shown in $>$ Table 3, EGCs with mixed type of histology had higher rates of pSM2 invasion $(P<0.001)$, higher risk of LVI $(P<0.001)$, higher rates of ulceration $(P<0.001)$, and lower rates of complete resection $(P<0.001)$ than pure differentiated or pure undifferentiated EGCs. With respect to the short-term outcomes of ESD, higher positive horizontal $(P<0.001)$ and vertical $(P<0.001)$ margins and lower complete resection rates $(P<0.001)$ were found in mixed-type EGCs compared with differentiated EGCs. To ascertain whether mixed-type EGC was an independent risk factor for LVI in incomplete resection of ESD, 
- Table2 Short-terms outcomes after endoscopic resection of EGCs.

\begin{tabular}{|c|c|}
\hline \multicolumn{2}{|l|}{ Characteristics } \\
\hline \multicolumn{2}{|l|}{ Indication based on pathological assessment } \\
\hline - Absolute indication & $238(41.8 \%)$ \\
\hline - Expanded indication & $271(47.5 \%)$ \\
\hline - Differentiated, pM, UL(-) & $236(87.1 \%)$ \\
\hline - Differentiated, pM, UL(+), $\leq 30$ mm & $3(1.1 \%)$ \\
\hline - Differentiated, pSM1, UL(-), $\leq 30 \mathrm{~mm}$ & $25(9.2 \%)$ \\
\hline - Undifferentiated, pM, UL(-), $\leq 20$ mm & $7(3.0 \%)$ \\
\hline - Beyond the expanded indication & $61(10.7 \%)$ \\
\hline \multicolumn{2}{|l|}{ Horizontal margin involvement } \\
\hline - Negative & $525(92.1 \%)$ \\
\hline - Positive & $43(7.5 \%)$ \\
\hline - Undetermined & $2(0.4 \%)$ \\
\hline \multicolumn{2}{|l|}{ Vertical margin involvement } \\
\hline - Negative & $562(98.6 \%)$ \\
\hline - Positive & $6(1.1 \%)$ \\
\hline - Undetermined & $1(0.2 \%)$ \\
\hline \multicolumn{2}{|l|}{ En bloc resection } \\
\hline - Yes & $547(96.0 \%)$ \\
\hline - No & $23(4.0 \%)$ \\
\hline \multicolumn{2}{|l|}{ Complete resection } \\
\hline - Yes & $520(91.2 \%)$ \\
\hline - No & $50(8.8 \%)$ \\
\hline \multicolumn{2}{|l|}{ Curability } \\
\hline - Curative resection & $473(83.0 \%)$ \\
\hline - Curative resection for absolute indication lesions & $230(48.6 \%)$ \\
\hline - Curative resection for expanded indication lesions & $243(51.4 \%)$ \\
\hline - Noncurative resection (overlapped) & $96(16.8 \%)$ \\
\hline - Positive resection margin & $47(49.0 \%)$ \\
\hline - Positive LVI & $16(17.7 \%)$ \\
\hline - Beyond Expanded indication & $61(63.5 \%)$ \\
\hline - $\mathrm{pSM2}$ & $32(33.3 \%)$ \\
\hline - Undifferentiated, >20 mm & $10(10.4 \%)$ \\
\hline - $\mathrm{UL}(+),>30 \mathrm{~mm}$ & $5(5.2 \%)$ \\
\hline - $\mathrm{pSM} 1,>30 \mathrm{~mm}$ & $10(10.4 \%)$ \\
\hline - Not completed & $9(1.5 \%)$ \\
\hline - Undetermined & $1(0.18 \%)$ \\
\hline \multicolumn{2}{|l|}{ Additional treatment for noncurative resection } \\
\hline - Close follow-up & $39(40.2 \%)$ \\
\hline - Repeated endoscopic resection & $16(16.5 \%)$ \\
\hline - Surgery & $42(43.3 \%)$ \\
\hline
\end{tabular}

- Table 2 (Continuation)

\begin{tabular}{|l|r|}
\hline \multicolumn{2}{|l|}{ Characteristics } \\
\hline Complication & $35(6.1 \%)$ \\
\hline - Bleeding & $5(14.3 \%)$ \\
\hline - Blood transfusion & $8(1.4 \%)$ \\
\hline - Perforation & $23(4.3 \%)$ \\
\hline - Fever & \\
\hline $\begin{array}{l}\text { pM, intramucosal; pSM1, depth of invasion < } 500 \text { um from the muscularis } \\
\text { mucosae; pSM2, depth of invasion } \geq 500 \text { um from the muscularis mucosae; } \\
\text { LVI, lymphatic vessel infiltration; UL, ulcer. }\end{array}$ \\
\hline
\end{tabular}

we performed a multivariate logistic regression including potential confounding factors with significant differences in univariate analysis (histology type, size of tumor, depth of invasion). As shown in Supplementary Table 1 and Supplementary Table 2, EGCs with mixed type of histology had a higher risk of LVI (OR: 1.2; $95 \% \mathrm{Cl}: 1.1-1.3 ; P<0.001)$ and incomplete resection rate (OR: $1.2 ; 95 \% \mathrm{Cl}: 1.0-1.3 ; P=0.005)$ compared with differentiated EGCs.

Duration of hospital stay after ESD was 6 days. Of the 570 ESDs performed, 35 patients $(6.1 \%)$ suffered bleeding requiring emergency endoscopic treatment and eight patients (1.4\%) experienced perforation requiring emergency endoscopic treatment. Among patients with bleeding, five (14.3\%) needed blood transfusion. In addition, 23 patients (4.3\%) had fever after ESD. All complications were managed without surgical intervention.

\section{Long-term outcomes of endoscopic submucosal dissection}

Three hundred fifteen patients were diagnosed from 2008 to 2015 but 13 patients were lost to follow-up and 302 patients were included for analysis with a median follow-up period of 54.1 months $(40.8,65.7)$. Five patients died during follow-up with one dying of gastric cancer and four of other causes. The patient who died of gastric cancer had EGC beyond the expanded indication group with non-curative resection and underwent additional surgery and had recurrent GC. The 3-year overall survival and cancer-specific survival were $99.4 \%$ and $100 \%$, respectively. The 5-year OS and CSS were $97.6 \%$ and $99.5 \%$, respectively.

As shown in Supplementary Table 3, a total of four patients (1.3\%) had recurrence of EGC, including two with an absolute indication and two with an expanded indication. > Fig. 2a shows the recurrence rate after ESD of EGCs, with 3-year and 5 -year recurrence rates of $0.7 \%$ and $1.2 \%$, respectively.

One patient $(4.4 \%, 1 / 23)$ with mixed-type EGC and three $(1.1 \%, 3 / 274)$ with pure EGC suffered recurrence during follow-up. Whether patients with mixed type histology had a higher risk of recurrence than those with differentiated EGC were then explored. As shown in > Fig. 3a, no difference in risk of recurrence $(P=0.32)$ was observed in patients with different histology types. In addition, the multivariate Cox regression model 
- Table 3 Short-term outcomes after endoscopic resection of EGCs according to the histological type.

\begin{tabular}{|c|c|c|c|c|c|c|}
\hline \multirow[t]{2}{*}{ Characteristics } & \multirow{2}{*}{$\begin{array}{l}\begin{array}{l}\text { Pure differ- } \\
\text { entiated }\end{array} \\
\mathrm{N}=\mathbf{5 2 0}\end{array}$} & \multirow{2}{*}{$\begin{array}{l}\begin{array}{l}\text { Pure undif- } \\
\text { ferentiated }\end{array} \\
\mathrm{N}=13\end{array}$} & \multicolumn{3}{|l|}{ Mixed type } & \multirow[t]{2}{*}{$P$ value $^{1}$} \\
\hline & & & $\begin{array}{l}\text { Total } \\
(\mathrm{N}=37)\end{array}$ & $\begin{array}{l}\text { Differentiated } \\
\text { predominantly } \\
(\mathrm{N}=26)\end{array}$ & $\begin{array}{l}\text { Poor differentiated } \\
\text { predominantly } \\
(\mathrm{N}=11)\end{array}$ & \\
\hline Size $(\mathrm{cm})$ & $18(10,26)$ & $22(20,27)$ & $25(18,38)$ & $25(20,38)$ & $20(15,40)$ & 0.001 \\
\hline \multicolumn{6}{|l|}{ Depth } & $<0.001$ \\
\hline - $\mathrm{pM}$ & $465(89.4 \%)$ & $11(84.6 \%)$ & $18(48.7 \%)$ & $13(50.0 \%)$ & $5(45.5 \%)$ & \\
\hline - pSM1 & $35(6.7 \%)$ & $2(15.4 \%)$ & $6(16.2 \%)$ & $4(15.4 \%)$ & $2(18.2 \%)$ & \\
\hline - pSM2 & $18(3.5 \%)$ & $0(0)$ & $11(29.7 \%)$ & $8(30.8 \%)$ & $3(29.7 \%)$ & \\
\hline - Undetermined & $1(0.2 \%)$ & $0(0)$ & $2(5.4 \%)$ & $1(3.8 \%)$ & $1(9.1 \%)$ & \\
\hline \multicolumn{6}{|l|}{ LVI status } & $<0.001$ \\
\hline - No & $512(98.5 \%)$ & $13(100 \%)$ & $28(75.7 \%)$ & $21(80.8 \%)$ & $7(63.6 \%)$ & \\
\hline - Yes & $7(1.4 \%)$ & $0(0)$ & $9(24.3 \%)$ & $5(19.2 \%)$ & $4(36.4 \%)$ & \\
\hline - Undetermined & $1(0.1 \%)$ & $0(0)$ & $0(0)$ & $0(0)$ & $0(0)$ & \\
\hline \multicolumn{6}{|l|}{ UL } & $<0.001$ \\
\hline - No & $512(98.5 \%)$ & $10(76.9 \%)$ & $34(91.9 \%)$ & $23(88.5 \%)$ & $11(100 \%)$ & \\
\hline - Yes & $8(1.5 \%)$ & $3(23.1 \%)$ & $3(8.1 \%)$ & $3(11.5 \%)$ & $0(0)$ & \\
\hline \multicolumn{6}{|l|}{ Horizontal margin involvement } & $<0.001$ \\
\hline - Negative & $486(93.5 \%)$ & $9(69.2 \%)$ & $30(81.1 \%)$ & $21(80.8 \%)$ & $9(81.8 \%)$ & \\
\hline - Positive & $32(6.2 \%)$ & $4(30.8 \%)$ & $7(18.9 \%)$ & $5(19.2 \%)$ & $2(18.2 \%)$ & \\
\hline - Undetermined & $2(0.4 \%)$ & $0(0)$ & $0(0)$ & $0(0)$ & $0(0)$ & \\
\hline \multicolumn{6}{|l|}{ Vertical margin involvement } & $<0.001$ \\
\hline - Negative & $516(99.2 \%)$ & $13(100 \%)$ & $33(89.2 \%)$ & $25(96.1 \%)$ & $8(72.7 \%)$ & \\
\hline - Positive & $2(0.4 \%)$ & $0(0)$ & $4(10.8 \%)$ & $1(3.9 \%)$ & $3(27.3 \%)$ & \\
\hline - Undetermined & $1(0.2 \%)$ & $0(0)$ & $0(0)$ & $0(0)$ & $0(0)$ & \\
\hline \multicolumn{6}{|l|}{ Complete resection } & $<0.001$ \\
\hline - Yes & $484(93.1 \%)$ & $9(69.2 \%)$ & $27(73.0 \%)$ & $20(76.9 \%)$ & $7(63.6 \%)$ & \\
\hline No & $36(6.9 \%)$ & $4(30.8 \%)$ & $10(27.0 \%)$ & $6(23.1 \%)$ & $4(36.4 \%)$ & \\
\hline \multicolumn{6}{|c|}{ Additional treatment for noncurative resection } & $<0.001$ \\
\hline - Close follow-up & $25(39.7 \%)$ & $5(45.4 \%)$ & $9(39.1 \%)$ & $8(50.0 \%)$ & 1 (14.3\%! & \\
\hline - Repeated endoscopic resection & $14(22.2 \%)$ & $1(9.1 \%)$ & $1(4.4 \%)$ & $1(6.2 \%)$ & $0(0)$ & \\
\hline - Surgery & $24(38.1 \%)$ & $5(45.5 \%)$ & $13(56.5 \%)$ & $7(43.8 \%)$ & $6(85.7 \%$ ! & \\
\hline
\end{tabular}

indicated that patients with a mixed-type EGC $(P=0.125)$ had no additional risk of recurrence compared with patients with differentiated EGCs after inclusion of potential confounding factors that had been shown to be significantly different by univariate analysis (curative resection status, location).

Thirteen patients (4.3\%) developed metachronous GC during follow-up, including five patients with an absolute indica- tion, seven with an expanded indication, and one patient beyond expanded indications. Fig. $\mathbf{2 b}$ displays the recurrence rate after ESD of EGCs, with 3-year and 5-year rates of MGC development of $2.7 \%$ and $4.2 \%$, respectively. No significant difference in risk of development of $\operatorname{MGC}(P=0.54$, $>$ Fig. $3 \mathbf{b})$ was found among patients with different histology types. With respect to Helicobacter pylori infection, five patients had $H$. pylori 

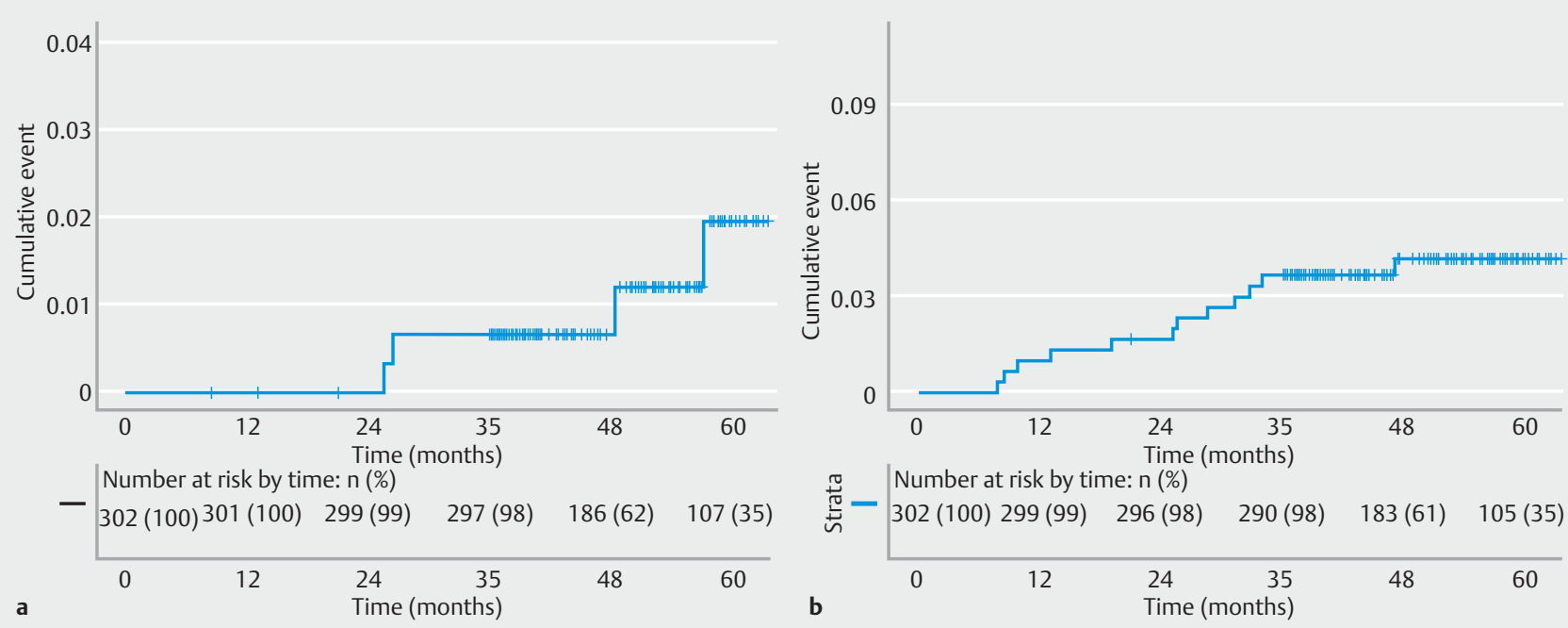

- Fig. 2 a Cumulative recurrence rate and b cumulative metachronous gastric cancer rate after endoscopic submucosal dissection of early gastric cancers.
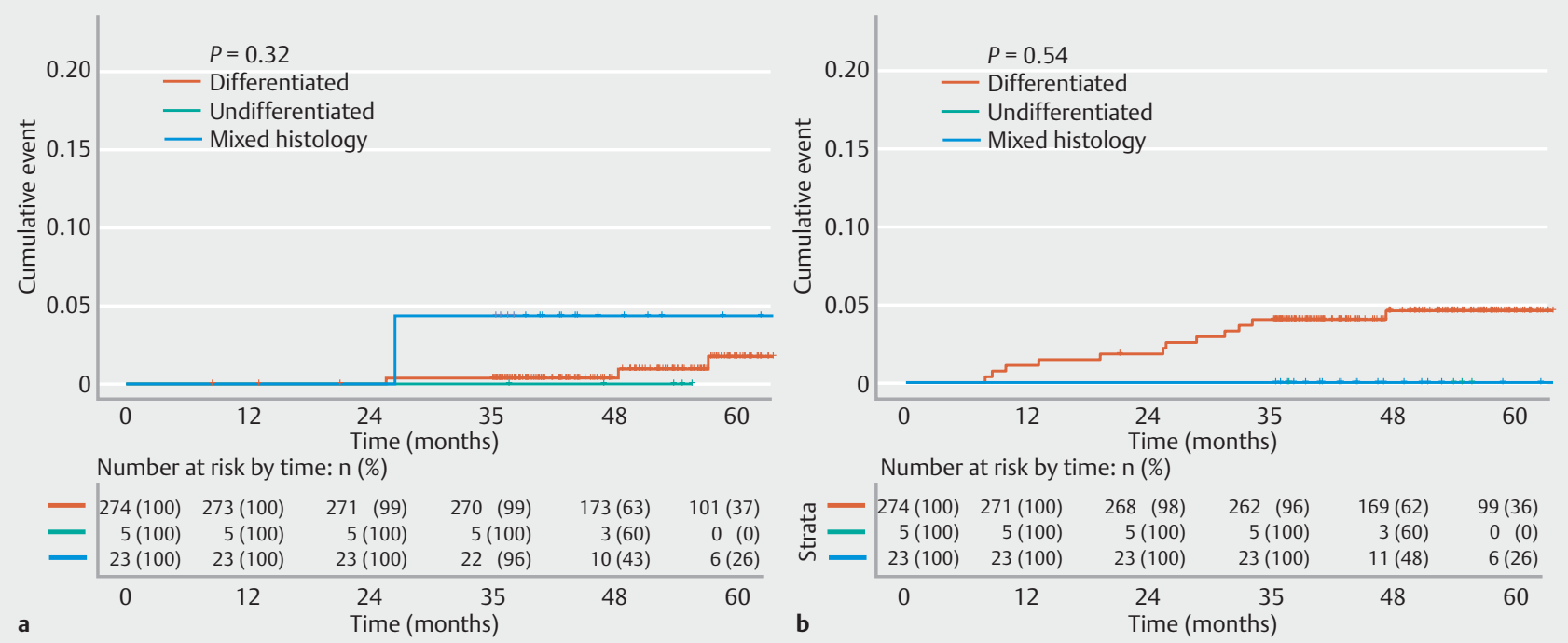

a Time (months)

Fig. 3 Comparison of a cumulative recurrence rate and $\mathbf{b}$ cumulative metachronous gastric cancer rate among patients with different histology types after endoscopic submucosal dissection of early gastric cancers.

infection before ESD of the first EGC successfully eradicated it. No patients who developed MGC had $H$. pylori infection.

\section{Discussion}

To the best of our knowledge, the present study is the first to evaluate short- and long-term outcomes of ESD of EGCs in China at a medical center with high-volumes of ESD involving 570 EGCs. It was demonstrated that curative ESD could be achieved in $96.2 \%$ of EGCs with absolute indication and $88.2 \%$ of EGCs with an expanded indication. With respect to long-term outcomes of ESD, $1.3 \%$ of patients experienced recurrence and $4.3 \%$ of patients developed MGC during endoscopic surveil- lance. The study also demonstrated that mixed-type EGC had a similar risk of recurrence to that of differentiated EGC, although higher risk of incomplete resection or LVI were observed in mixed-type EGC compared with differentiated EGC.

After the use of ESD in the treatment of EGCs for more than 10 years, there have been increasing numbers of studies of short- and long-term outcomes of ESD for EGC, from Japan [2, 22-29], Korea [30-32] and Western countries [4-6, 33, 34] ( $\triangleright$ Table 4). It has been reported that ESD achieved en bloc resection rates of $79 \%$ to $100 \%$, complete resection of $64 \%$ to $100 \%$, and curative resection of $73.6 \%$ to $90.2 \%$ in EGCs fulfilling absolute or expanded indications in Western countries [4$6,33,34]$. En bloc rates of $95.3 \%$ to $99.2 \%$, complete resection 


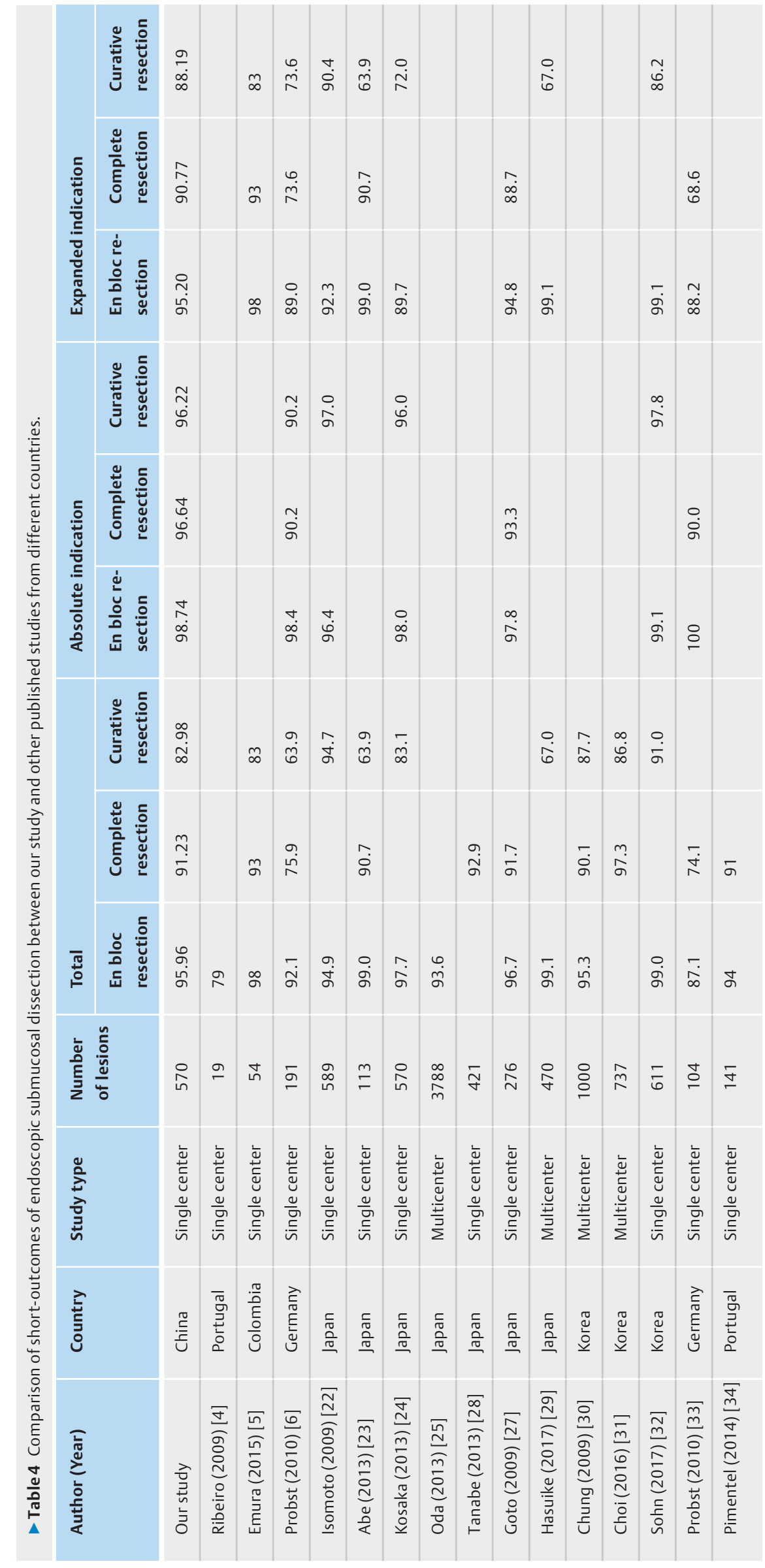


of $84.6 \%$ to $97.8 \%$ and curative resection of $86.8 \%$ [31] in Korea [30-32], and en bloc rates of $92.7 \%$ to $94.9 \%$, complete resection of $86 \%$ to $91.7 \%$, and curative resection in $73.6 \%$ to $94.7 \%$ in Japan [2, 22-29] have also been reported. Rates of en bloc resection, complete resection, and curative resection were found to be $96.0 \%, 91.2 \%$, and $83.0 \%$ overall, $98.7 \%, 96.6 \%$ and $96.2 \%$ for an absolute indication, and $95.2 \%, 90.8 \%$, and $88.2 \%$ for an expanded indication, respectively, in our medical center. The resection rates were comparable to those in Japan and Korea, indicating that Japanese indications for ESD could be applied in China [9].

Western countries reported bleeding rates of $6.3 \%$ to $8 \%$ and perforation of $1.1 \%$ to $4 \%[5,6,34]$, with Japan and Korea reporting bleeding rates of $6 \%$ to $15.6 \%$ and perforation rates of $1.2 \%$ to $8.2 \%$ [32,35]. The complication data quoted above were comparable to that observed in the present study. We confirmed the safety of ESD with $6.14 \%$ of patients experiencing bleeding and $1.40 \%$ perforation, both of which were managed endoscopically.

In the present study, scheduled follow-up identified four patients $(1.32 \%)$ in whom recurrence was experienced over a follow-up time of at least 36 months. Compared with previous recurrence rate data ranging from $0.2 \%$ to $1.4 \%$ after curative resection in Asian countries [2, 22-29, 32, 36], patients treated in the present study had a similar recurrence rate of $1.32 \%$, but lower than that of a study from a Western country in which $4.8 \%$ of patients experienced recurrence. In the present study, to identify the potential reason for recurrence after curative resection, we checked and reexamined the pathology of ESD specimens from the four patients and that all of them had lowgrade intraepithelial neoplasia at the resection margin and were recorded as having a negative resection margin with a recommendation for follow-up. Meanwhile, the possibility of MGC occurring at the site or very close site of the prior ESD could not be neglected due to the pulling surrounding normal mucosa of scar. In addition, patients had high 5-year OS of $97.6 \%$ and CSS of $99.5 \%$ in the present study, comparable to in other Asian countries [22-29, 32].

Unlike surgery, ESD enables both curative resection and preservation of the entire stomach, which increases the risk of developing MGC. The incidence rate for MGC varied among studies, from $2.8 \%$ to $15.9 \%$ [6, 22-26, 29, 32]. In the present study, 13 patients (4.3\%) developed MGCs, all of which were resected curatively through a second ESD, with no impact on survival. When we analyzed these 13 patients, we found that $46.2 \%$ (6/ 13) developed MGC in the third year after endoscopic resection of EGC: three in the first year, two patients in the second year, and two after more than 3 years. This finding may indicate that regular follow-up should be performed no matter how long it has been since the first EGC was resected. Thus, strict endoscopic follow-up is required for early detection of MGCs. All MGCs in the present study were diagnosed as EGCs, confirming the rationale of the follow-up schedule. However, occurrence rate of MGC should be interpreted with caution since only small number of patients with 3-year follow up were included and the occurrence rate of MGC may be higher after analyzing 5-year MGC occurrence rate with enough number of patients.
A number of studies observed a higher risk of incomplete resection in mixed-type EGC compared with pure EGC [17-19], which was also confirmed in the present study, in which only $73.0 \%$ of complete resection could be achieved in mixed-type EGC and even lower complete resection (63.6\%) in MU-EGC. Two studies reported controversial results regarding the recurrence rate of mixed-type EGC compared with pure EGC. Min et al. [19] reported comparable recurrence but Han et al. [17] reported mixed-type EGC was an independent risk factor for recurrence. This contradictory conclusion can be explained because Min et al. [19] compared risk of recurrence in patients with curative resection of ESD but Han et al. [17] also included patients with noncurative resection. In the present study, only one patient with mixed-type EGC experienced recurrence and we found no significant risk of a higher rate of recurrence in mixed-type EGC, although mixed-type EGC was an independent risk factor for LVI and incomplete resection. Our study further indicated that mixed-type EGC may not be a risk factor for higher risk of recurrence in guarantee of complete resection. However, higher risk of incomplete resection in mixed-type EGC was observed in multiple studies [17-29] and the present study, indicating that the resection margin should be carefully examined and be larger than pure EGC to achieve complete resection for mixed-type EGC. Further studies should be performed for validation of our finding.

The study was subject to a number of limitations. First, selection bias was inevitable because it was retrospective and from a single medical center. Second, the present study was conducted at a single major medical cancer center in a large metropolitan region of China, which employs many highly experienced endoscopists with specific expertise in ESD. Thus, a prospective multicenter study may more reliably measure the effectiveness of ESD for the treatment of EGCs in China including short- and long-term outcomes of ESD. Third, a limited number of mixed-type EGCs were included in the analysis. The results of recurrence or development of MGCs after ESD for mixed-type EGC should be further confirmed through analysis of a large number of lesions. Fourth, only 3-year long-term outcomes, including the incidence rate for MGC, were investigated. Careful attention must be paid to evaluating the outcomes of MGC in this study because the follow-up period was $<5$ years and the number of patients was small.

\section{Conclusions}

In summary, the present study confirmed the effectiveness of ESD for treatment of EGCs with satisfactory short- and longterm outcomes in China. To the best of our knowledge, this study is the first and largest on effectiveness and follow-up data from ESD for EGCs in China. Scheduled endoscopic surveillance should be conducted after ESD, especially for patients with noncurative ESD and to achieve no additional treatment from high local recurrence, and a rate for MGC after ESD of about $4.3 \%$. In addition, patients with mixed histology experienced a similar rate of recurrence and development of MGC compared with those who had pure differentiated EGC, al- 
though a higher risk of incomplete ESD was experienced in patients with mixed histology.

\section{Acknowledgements}

This work was supported by grants from the National Natural Science Foundation of China (No. 82070573), the Shanghai Municipal Education Commission: Gaofeng Clinical Medicine grant support (No. DLY201501), Cross Medical Research Fund of Translational Medicine, Shanghai jiao Tong University (No. ZH2018ZDA06), Shanghai Municipal Key Clinical Specialty (No. shslczdzk05902), and Shanghai Municipal Commission of Health and Family Planning (No. 2018YQ29), Program for Promoting Advanced Appropriate Technology of Shanghai Health Commission (2019SY003), and Health Technology Projects of Pudong New Area (PW2020D-12).

\section{Competing interests}

The authors declare that they have no conflict of interest.

\section{References}

[1] Oda I, Saito D, Tada M et al. A multicenter retrospective study of endoscopic resection for early gastric cancer. Gastric Cancer 2006; 9: 262-270

[2] Tanabe S, Hirabayashi S, Oda I et al. Gastric cancer treated by endoscopic submucosal dissection or endoscopic mucosal resection in Japan from 2004 through 2006: JGCA nationwide registry conducted in 2013. Gastric Cancer 2017; 20: 834-842

[3] Lian J, Chen S, Zhang Y et al. A meta-analysis of endoscopic submucosal dissection and EMR for early gastric cancer. Gastrointest Endosc 2012; 76: 763-770

[4] Dinis-Ribeiro M, Pimentel-Nunes P, Afonso M et al. A European case series of endoscopic submucosal dissection for gastric superficial lesions. Gastrointest Endosc 2009; 69: 350-355

[5] Emura F, Mejia J, Donneys A et al. Therapeutic outcomes of endoscopic submucosal dissection of differentiated early gastric cancer in a Western endoscopy setting (with video). Gastrointest Endosc 2015; 82: 804-811

[6] Probst A, Schneider A, Schaller T et al. Endoscopic submucosal dissection for early gastric cancer: are expanded resection criteria safe for Western patients? Endoscopy 2017; 49: 855-865

[7] Abdelfatah MM, Barakat M, Lee $\mathrm{H}$ et al. The incidence of lymph node metastasis in early gastric cancer according to the expanded criteria in comparison with the absolute criteria of the Japanese Gastric Cancer Association: a systematic review of the literature and meta-analysis. Gastrointest Endosc 2018; 87: 338-347

[8] Choi AH, Nelson RA, Merchant SJ et al. Rates of lymph node metastasis and survival in T1a gastric adenocarcinoma in Western populations. Gastrointest Endosc 2016; 83: 1184-1192 e1181

[9] Japanese Gastric Cancer A. Japanese gastric cancer treatment guidelines 2010 (ver. 3). Gastric Cancer 2011; 14: 113-123

[10] Song WC, Qiao XL, Gao XZ. A comparison of endoscopic submucosal dissection (ESD) and radical surgery for early gastric cancer: a retrospective study. World J Surg Oncol 2015; 13: 309

[11] Chiu PW, Teoh AY, To KF et al. Endoscopic submucosal dissection (ESD) compared with gastrectomy for treatment of early gastric neo- plasia: a retrospective cohort study. Surg Endosc 2012; 26: 35843591

[12] Fu QY, Cui Y, Li XB et al. Relevant risk factors for positive lateral margin after en bloc endoscopic submucosal dissection for early gastric adenocarcinoma. J Dig Dis 2016; 17: 244-251

[13] Zhang Y, Huang L, Li L et al. Endoscopic submucosal dissection for early gastric neoplasms in elderly patients. J Laparoendosc Adv Surg Tech A 2014; 24: 391-398

[14] Park HK, Lee KY, Yoo MW et al. Mixed Carcinoma as an Independent Prognostic Factor in Submucosal Invasive Gastric Carcinoma. J Korean Med Sci 2016; 31: 866-872

[15] Seo HS, Lee GE, Kang MG et al. Mixed Histology Is a Risk Factor for Lymph Node Metastasis in Early Gastric Cancer. J Surg Res 2018; 236: 271-277

[16] Takizawa K, Ono H, Kakushima N et al. Risk of lymph node metastases from intramucosal gastric cancer in relation to histological types: how to manage the mixed histological type for endoscopic submucosal dissection. Gastric Cancer 2013; 16: 531-536

[17] Han JP, Hong SJ, Kim HK. Long-term outcomes of early gastric cancer diagnosed as mixed adenocarcinoma after endoscopic submucosal dissection. J Gastroenterol Hepatol 2015; 30: 316-320

[18] Horiuchi Y, Fujisaki J, Yamamoto N et al. Mixed poorly differentiated adenocarcinoma in undifferentiated-type early gastric cancer predicts endoscopic noncurative resection. Gastric Cancer 2018; 21 : 689-695

[19] Min BH, Kim KM, Park CK et al. Outcomes of endoscopic submucosal dissection for differentiated-type early gastric cancer with histological heterogeneity. Gastric Cancer 2015; 18: 618-626

[20] Japanese Gastric Cancer A. Japanese classification of gastric carcinoma: 3rd English edition. Gastric Cancer 2011; 14: 101-112

[21] Kim WH, Park CK, Kim YB et al. A Standardized Pathology Report for Gastric Cancer. The Korean Journal of Pathology 2005; 39: 106-113

[22] Isomoto H, Shikuwa S, Yamaguchi $\mathrm{N}$ et al. Endoscopic submucosal dissection for early gastric cancer: a large-scale feasibility study. Gut 2009; 58: 331-336

[23] Abe S, Oda I, Suzuki H et al. Short- and long-term outcomes of endoscopic submucosal dissection for undifferentiated early gastric cancer. Endoscopy 2013; 45: 703-707

[24] Kosaka T, Endo M, Toya Y et al. Long-term outcomes of endoscopic submucosal dissection for early gastric cancer: a single-center retrospective study. Dig Endosc 2014; 26: 183-191

[25] Oda I, Oyama T, Abe S et al. Preliminary results of multicenter questionnaire study on long-term outcomes of curative endoscopic submucosal dissection for early gastric cancer. Dig Endosc 2014; 26 : 214-219

[26] Tanabe S, Ishido K, Higuchi K et al. Long-term outcomes of endoscopic submucosal dissection for early gastric cancer: a retrospective comparison with conventional endoscopic resection in a single center. Gastric Cancer 2014; 17: 130-136

[27] Goto O, Fujishiro M, Kodashima S et al. Outcomes of endoscopic submucosal dissection for early gastric cancer with special reference to validation for curability criteria. Endoscopy 2009; 41: 118-122

[28] Tanabe S, Ishido K, Matsumoto T et al. Long-term outcomes of endoscopic submucosal dissection for early gastric cancer: a multicenter collaborative study. Gastric Cancer 2017; 20: 45-52

[29] Hasuike N, Ono H, Boku N et al. A non-randomized confirmatory trial of an expanded indication for endoscopic submucosal dissection for intestinal-type gastric cancer (cT1a): the Japan Clinical Oncology Group study (JCOG0607). Gastric Cancer 2018; 21: 114-123

[30] Chung IK, Lee JH, Lee SH et al. Therapeutic outcomes in 1000 cases of endoscopic submucosal dissection for early gastric neoplasms: Korean ESD Study Group multicenter study. Gastrointest Endosc 2009; 69: $1228-1235$ 
[31] Choi IJ, Lee NR, Kim SG et al. Short-Term outcomes of endoscopic submucosal dissection in patients with early gastric cancer: a prospective multicenter cohort study. Gut Liver 2016; 10: 739-748

[32] Sohn SH, Lee SH, Kim KO et al. Therapeutic outcomes of endoscopic submucosal dissection for early gastric cancer: single-center study. Eur J Gastroenterol Hepatol 2017; 29: 61-67

[33] Probst A, Pommer B, Golger D et al. Endoscopic submucosal dissection in gastric neoplasia - experience from a European center. Endoscopy 2010; 42: 1037-1044
[34] Pimentel-Nunes P, Mourao F, Veloso N et al. Long-term follow-up after endoscopic resection of gastric superficial neoplastic lesions in Portugal. Endoscopy 2014; 46: 933-940

[35] Saito I, Tsuji Y, Sakaguchi Y et al. Complications related to gastric endoscopic submucosal dissection and their managements. Clin Endosc 2014; 47: 398-403

[36] Suzuki H, Oda I, Abe S et al. High rate of 5-year survival among patients with early gastric cancer undergoing curative endoscopic submucosal dissection. Gastric Cancer 2016; 19: 198-205 